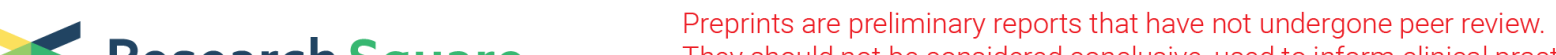 Research Square They should not be considered conclusive, used to inform clinical practice, or referenced by the media as validated information.
}

\section{A Facilitated Gas Transport Strategy: 3D Porous Hyper-crosslinked Polymers Incorporating 6FDA- based Polyimide Matrix for the Design and Fabrication of Mixed Matrix Membranes with High Permeability}

\section{Zan Chen}

Hebei University of Technology

\section{Longfei Peng}

Hebei University of Technology

Yinhui Li ( $\nabla$ liyinhui@hebut.edu.cn )

Hebei University of Technology

\section{Weixin Li}

Hebei University of Technology

\section{Cuijia Duan}

China National Offshore Oil Corporation Tianjin Chemical Research \& Design Institute

\section{Biao Yuan}

China National Offshore Oil Corporation Tianjin Chemical Research \& Design Institute

\section{Shuo Yan}

China National Offshore Oil Corporation Tianjin Chemical Research \& Design Institute

\section{Research Article}

Keywords: 6FDA-based polyimide, hyper-crosslinking polymers, gas transport, molecular dynamic simulations

Posted Date: January 28th, 2021

DOI: https://doi.org/10.21203/rs.3.rs-151228/v1

License: (c) (i) This work is licensed under a Creative Commons Attribution 4.0 International License. Read Full License 


\section{Abstract}

Economic membrane technologies are a perennial hot topic in the field of natural gas purification and $\mathrm{O}_{2}$ enrichment. In this work, novel hyper cross-linked polymers (HCPs) incorporating 6-FDA based polyimide (PI) MMMs were prepared via a casting method for enhancing different gas transport $\left(\mathrm{CO}_{2}, \mathrm{CH}_{4}, \mathrm{O}_{2}\right.$, and $\mathrm{N}_{2}$ ). The gas permeation experiments showed that compared with pure PI films, adding HCPs effectively promotes gas transport, increases gas permeability, and maintains ideal selectivity. The permeability of $\mathrm{HCPS} / \mathrm{PI}$ MMMs toward $\mathrm{CO}_{2}$ and $\mathrm{O}_{2}$ was as high as 105.85 barrer and 24.03 barrer, respectively, and the ideal selectivity of $\mathrm{CO}_{2} / \mathrm{CH}_{4}$ and $\mathrm{O}_{2} / \mathrm{N}_{2}$ was 15.67 and 3.00 , respectively. Molecular dynamic simulations further verified that adding HCPs was beneficial to gas transport thanks to large FFV of MMMs. Thus, HCPs have potential utility in the fabrication of MMMs for facilitating gas transport in the field of natural gas purification and $\mathrm{O}_{2}$ enrichment.

\section{Introduction}

Gas separation is an essential separation process in natural gas purification, flue gas separation, and $\mathrm{O}_{2}$ enrichment ${ }_{[1-6]}$. Membrane technologies are usually used for gas separation thanks to its environmentally friendly process and low energy consumption[7-9]. Unfortunately, the permeation has always been a problem restricting gas separation performance in membrane technology[10]. In general, gas separation membranes are divided into homogeneous and heterogeneous structures. Dense membranes with homogeneous structures have poor permeation and thus are restricted in industrial applications.

Mixed matrix membranes (MMMs) with heterogeneous structures are excellent candidates for gas separation because they facilitate gas transport of fillers, including plenty of porous structures and functional groups[11]. Porous structures promote gas transport and functional groups and are beneficial to gas dissolution-diffusion, which break through low permeation and realize highly effective gas separation[12-14].

MMMs consist of fillers with porous structures in a polymeric matrix, including rubber polymers and glassy polymers. Normally, glassy polymers such as polysulfone[15, 16], cellulose[17, 18], and polyimide[19-21] can nicely separate well gas. Of these, polyimide (PI) with highly thermal stability, good chemical stability, and strong mechanical stability has been extensively used as gas separation membrane[22, 23]. More significantly, the largely fractional free volume (FFV) of aromatically fluorinated $\mathrm{PI}$ is beneficial to gas transport[24, 25]. However, a polymer matrix with a large FFV will result in gas selectivity reduction that falls into conventional trade-off effects. Thus, preparing PI MMMs with high permeability and suitable selectivity is still an important challenge.

In recent years, researchers have focused on the utility of fillers with tailored porous structure and functional groups for improving gas transport and overcoming trade-off effect. For example, Koros and coworkers synthesized zeolite-like MOF (ZMOF) filler for the preparation of ZMOF/PI MMMs. Gas 
permeation experiments show that ZMOF/PI MMMs enhanced $\mathrm{CO}_{2}$ permeability due to the incorporation of ZMOF filler with an affinity $\mathrm{CO}_{2}$ framework that enhanced $\mathrm{CO}_{2}$ sorption and facilitated gas diffusivity[26]. Yeong and coworkers prepared zeolite T as inorganic filler for the fabrication of Zeolite T/PI MMMs in $\mathrm{CO}_{2} / \mathrm{CH}_{4}$ separation. Zeolite $\mathrm{T}$ could effectively improve $\mathrm{CO}_{2}$ and $\mathrm{CH}_{4}$ transport properties. Compared to pure PI films, the permeability of Zeolite T/PI MMMs toward $\mathrm{CO}_{2}$ increased $80 \%$. Additionally, Zeolite T/PI MMMs also improved in $\mathrm{CO}_{2}$ plasticization resistant[27]. Chung and coworkers fabricated zeolitic imidazolate framework (ZIF)-71 inorganic fillers with a diameter of less than $100 \mathrm{~nm}$, and the fillers were compatibly incorporated in PI membranes. ZIF-71 in the PI membranes enhanced gas permeation, and the permeability of ZIF-71/PI MMMs toward pure $\mathrm{CO}_{2}$ was three-fold higher than pure PI membrane[28]. In short, developing a perfect filler in MMMs is an effective approach to promote gas transport and break through the trade-off effect.

To date, different fillers, such as silica nanoparticles[29], COFs[30], MOFs[31, 32], ZIFs[33, 34], and carbon nanotubes[35,36] have been used to prepare polyimide MMMs and enhance permeability and selectivity. The permeability and selectivity of MMMs are greatly improved after incorporating the fillers. However, the incompatible interfaces between fillers and polymer matrix have always been a problem due to the effect of defects caused by weak compatibility on gas transport and selectivity[37]. Recently, hypercrosslinking polymers (HCPs) with porous organic frameworks have become a hot issue. HCPs usually exhibit a free-defect interface in the course of fabrication of MMMs thanks to good compatibility between the fillers and polymer matrix. More importantly, HCPs are a kind of novel filler with fine prospect in MMMs due to their good ability to capture gas[38]. Some reports indicated that the use of HCPs for the preparation of MMMs can break through the trade-off effect and greatly facilitate gas transport[39]. However, there are limited reports on the use of HCPs for the preparation of HCPs/PI MMMs, and there are few studies on the gas transport of HCPs/PI MMMs. Clearly explaining the gas transport in HCPs/PI MMMs has an important impact on the structural design and manufacturing process of PI MMMs.

In this work, we first prepared 6FDA-based PI MMMs using novel HCPs as the organic filler. We then used 2-phenylimidazole and a, a-dichloro-p-xylene as reaction monomers, and the HCPs were synthesized by direct polymerization. Poly(2-phenylimidazole-co-a, a-dichloro-p-xylene) is simultaneously a weak acid and a weak basic due to its special chemical structure. HCPs and 6FDA-based PI were blended to prepare the HCPs/PI MMMs by a solution casting process. The gas separation properties and molecular dynamic simulations of the HCPs/PI MMMs are researched by separation of $\mathrm{CO}_{2} / \mathrm{CH}_{4}$ and $\mathrm{O}_{2} / \mathrm{N}_{2}$.

\section{Experimental}

\subsection{Materials and chemicals}

Hexafluoroisopropylidene diphthalic anhydride (6FDA), diaminophyenylindane (DAPI), dimethylacetamide (DMAc), acetic anhydride, trimethylamine (TEA), 2-phenylimidazole, $a, a^{\prime}$-dichloro-p-xylene, 1,2dichloroethane, anhydrous ferric chloride $\left(\mathrm{FeCl}_{3}\right)$, and methanol were analytical grade and used without 
further purification. Nitrogen $\left(\mathrm{N}_{2}\right)$, methane $\left(\mathrm{CH}_{4}\right)$, carbon dioxide $\left(\mathrm{CO}_{2}\right)$, and oxygen $\left(\mathrm{O}_{2}\right)$ are high purity and were purchased from Tianjin West Development Co., Ltd. Distilled water was made from a homemade distillation device.

\subsection{Preparation of PI MMMs}

\section{Synthesis of hyper-crosslinked polymers (HCPs)}

The synthetic route of HCPs refers to the literature $[40,41]$. The process is as follows: 5 mmol of 2phenylimidazole and $5 \mathrm{mmol}$ of $a$, $\mathrm{a}^{\prime}$-dichloro-p-xylene were fully dissolved in $10.00 \mathrm{ml}$ of 1,2dichloroethane, and then $10 \mathrm{mmol}$ of $\mathrm{FeCl}_{3}$ was added into the above solution. The system was polymerized at $80^{\circ} \mathrm{C}$ for $24 \mathrm{~h}$ under a nitrogen atmosphere. The resulting brown product was stirred in methanol for $24 \mathrm{~h}$ to remove unreacted monomers and anhydrous $\mathrm{FeCl}_{3}$. After the product was filtered and washed with ultrapure water, the product was dried in a vacuum oven at $60^{\circ} \mathrm{C}$ for $12 \mathrm{~h}$. The final product was called HCPs. The synthetic process is shown in Scheme 1. HCP characterization is shown in Figure S1. Figure S1 shows that HCPs are amorphous with a diameter of about 102 nm.

\section{Preparation of PI MMMs}

The HCPs were added to $5.0 \mathrm{ml}$ of DMAc solution and stirred for $2 \mathrm{~h}$ (the mass ratio of $\mathrm{HCPs}$ and $\mathrm{PI}$ is $0.02,0.04,0.06$, and 0.08 ). We then continued dispersing for another $1 \mathrm{~h}$ by ultrasonic treatment after $0.500 \mathrm{~g}$ of $\mathrm{PI}$ was added into the above system and stirred for $12 \mathrm{~h}$ to totally dissolve. This process was according to Freeman[42], and the synthesis process of 6FDA-based PI is shown in Scheme S1. The casting solution was evenly coated on an ultra-flat watch glass, and DMAc was evaporated at $60^{\circ} \mathrm{C}$. Finally, the formed membrane was laid on a common watch glass to heat treatment at $60^{\circ} \mathrm{C}$ for $2 \mathrm{~h}$, $120^{\circ} \mathrm{C}$ for $4 \mathrm{~h}, 180^{\circ} \mathrm{C}$ for $4 \mathrm{~h}$, and $220^{\circ} \mathrm{C}$ for $12 \mathrm{~h}$. The PI MMMs were obtained and labeled as HCPs0.02/PI, HCPs-0.04/PI, HCPs-0.06/PI, and HCPs-0.08/PI. (A blank experiment with pure PI film was prepared via the steps aforementioned except for adding HCPs.)

\subsection{Characterization}

The crystalline phase of HCPs and HCPs/PI MMMs was determined by an X-ray diffractometer (XRD, Bruker D8 Discover, German). After grinding the powders with an agate mortar, the powders were put on a glass slide and tested at $10 \% \mathrm{~min}$ sweep speed with a Cu target. The tube voltage, the current, and the scan range were $40 \mathrm{kV}, 200 \mathrm{~mA}$, and $5^{\circ}-50^{\circ}$, respectively. The chemical structures of HCPs and HCPs/PI MMMs were characterized by Fourier transform infrared spectroscopy (FT-IR, Bruker Vertex 70, German). The test range of HCPs is $4000-600 \mathrm{~cm}^{-1}$, which was tested by a $\mathrm{KBr}$ tablet method. The test range of MMMs is $4000-600 \mathrm{~cm}^{-1}$ via reflection mode test. The morphology of HCPs and HCPs/PI MMM adhered 
to the carbon conductive adhesive and was sprayed with gold and detected by a scanning electron microscope (SEM, Hitachi S4800, Japan). $\mathrm{N}_{2}$ adsorption was used to measure the specific surface area and pore size distribution of HCPs (BET and BJH, micromeritics ASAP2460, USA). The mechanical strength of the HCPs/PI MMMs was tested using a universal stretching machine (XD-121A, Shanghai Xinda Instrument Co., Ltd., China). The tested membranes were cut into $6 \mathrm{~cm} \times 1 \mathrm{~cm}$ strips to test their tensile strength and elongation at breaking.

\subsection{Evaluation of gas separation performance}

Single gas permeation performances of pure PI film and HCPs/PI MMMs were measured by the variablevolume method. After these membranes were placed into the porous supporter, the system was sealed in a specific membrane chamber. The feed gas pressure was conveniently adjusted by a back-pressure gauge. The gas permeability was determined in the sequence of $\mathrm{CH}_{4}, \mathrm{~N}_{2}, \mathrm{O}_{2}$, and $\mathrm{CO}_{2}$ at $308.15 \mathrm{~K}$ under $0.1 \mathrm{MPa}$ with an effective membrane area of approximately $\pi \mathrm{cm}^{2}$. The frame cell was degassed for $1 \mathrm{~h}$ to remove the residual air. The pressure on the lower side of membrane chamber was controlled at -0.1 $\mathrm{MPa}$. The experimental data were recorded when the system was kept at a stable state.

Permeability ( $P$, barrer) was the averaged value from three times experimental results. According to the growing rate of downstream pressure with time under steady state, $P$ was calculated by Eq. (1) as below:

$$
P=\frac{273 \times 10^{10}}{760} \frac{V l}{A T\left(p_{2} \times \frac{76}{14.7}\right)} \frac{d_{p}}{d_{t}} .
$$

Here, $V$ is the gas volume under the membrane, $A$ is the effective membrane area $\left(\mathrm{cm}^{2}\right), T$ is the experimental temperature $(K), I$ is the thickness of membrane $(\mathrm{cm})$, and $p_{2}$ is the upper pressure of membrane.

The ideal selectivity for two gases is calculated via Eq. (2):

\section{$\mathrm{a}_{\mathrm{A} / \mathrm{B}}=P_{A} / P_{B}$}

Eq. (2)

Here, $P_{A}$ is the gas permeability of pure gas $\mathrm{A}$, and $P_{B}$ is the gas permeability of pure gas $\mathrm{B}$.

\subsection{Molecular dynamic simulations}

The FFV of PI film and HCPs/PI MMMs was calculated by molecular dynamic (MD) simulation. Gromacs 2018.4 package was used to perform all molecular models for MD simulation. In detail, twenty-three 
molecular chains composed of dianhydride and diamine with 30 repeating units (including a big HCPs unit in MMMs) for each were placed in a cubic simulation cell and make the best use of the balance between cell dimension and calculation time. Energy minimization processes over 800 iterations to obtain stable structures were used to perform all of the molecular models. Then, the MD calculations were carried out under an NVT ensemble for duration of $100 \mathrm{ps}$ at $300 \mathrm{~K}$. The Newton second law of motion was applied to estimate the dynamic behaviors of polymer molecules. The theoretical calculations adopted the COMPASS force field. The FFV of PI film and HCPs/PI MMMs can be calculated by the following Eq. (3):

\section{$F F V=\left(V_{0}-V_{1}\right) / V_{0}=\left(V-1.3 V_{2}\right) / V_{0}$}

\section{Eq. (3)}

Here, $V_{0}, V_{1}$ and $V_{2}$ are the specific volume, occupied volume and the van der Waals volume of 6FDA based polyimide, respectively.

\section{Results And Discussion}

\subsection{SEM images of PI membranes}

Whether HCPs can be uniformly dispersed in MMMs is an important indicator for evaluating the quality of MMMs. Gas separation performance will greatly reduce once the HCPs get together and form agglomeration. Fig. $1 \mathrm{a}$ and $1 \mathrm{~b}$ show the overlooking SEM image and the cross-sectional SEM image of pure PI film, respectively. A pure PI film with a homogenous structure is relatively smooth and dense. Fig. $1 \mathrm{c}, 1 \mathrm{e}, 1 \mathrm{~g}$, and $1 \mathrm{i}$ show the surface SEM images of HCPs-0.02/PI MMMs, HCPs-0.04/PI MMMs, HCPs$0.06 / \mathrm{PI}$ MMMs, and HCPs-0.08/PI MMMs, respectively. The results show that the amount of HCPs in MMMs increases with continued addition of HCPs. When the amount of HCPs is $0.08 \mathrm{wt}$.\%, the size of HCPs becomes relatively larger because slight agglomeration occurs due to many more HCPs. Fortunately, HCPs have no large-scale agglomeration and are still well dispersed in PI films indicating good compatibility between HCPs and PI films[29].

Fig. $1 \mathrm{~d}, 1 \mathrm{f}, 1 \mathrm{~h}$, and $1 \mathrm{j}$ show cross-sectional SEM images of HCPs-0.02/PI MMMs, HCPs-0.04/PI MMMs, HCPs-0.06/PI MMMs, and HCPs-0.08/PI MMMs, respectively. The distribution and sizes of HCPs agree with the SEM images. All SEM images show that the as-prepared HCPs/PI MMMs have no defects after adding HCPs, which can avoid side leakage during gas separation and provide a better guarantee for the next step of gas separation.

\subsection{FT-IR spectra of PI membranes}

FT-IR spectra can assess the degree of amidization of the polymer. The FT-IR spectra of pure PI film and different HCPs/PI MMMs are shown in Fig. 2. The wavenumber at $1785 \mathrm{~cm}^{-1}$ and $1725 \mathrm{~cm}^{-1}$ is the symmetric and asymmetric stretching vibration peaks of $\mathrm{C}=\mathrm{O}$ in the imides groups, while the 
wavenumber at $1370 \mathrm{~cm}^{-1}$ and $718 \mathrm{~cm}^{-1}$ is the stretching and bending vibration peaks of $\mathrm{C}-\mathrm{N}[43,44]$. The characteristic peak at about $3300-3500 \mathrm{~cm}^{-1}$ is the stretching vibration of $\mathrm{N}-\mathrm{H}$ in poly(amic acid), which is not detected. The result shows that the polymers achieved amidation. In addition, the assigned peaks at $1250 \mathrm{~cm}^{-1}$ and $1140 \mathrm{~cm}^{-1}$ are the stretching vibration of C-F in $\mathrm{PI}$, demonstrating that the fluorinated polyimides have been successfully obtained[45]. The characteristic peaks of different HCPs/PI MMMs are the same as those of pure PI film, indicating that no new chemical bond was formed between HCPs and PI.

\subsection{Mechanical strength of PI membranes}

The MMMs need to withstand a certain pressure during gas separation. Therefore, the MMMs should have sufficient mechanical strength. The mechanical strength of HCPs/PI MMMs is necessarily assessed. Elongation at breaking and the tensile strength of pure $\mathrm{PI}$ film and different $\mathrm{PI} / \mathrm{HCPs} \mathrm{MMMs}$ are displayed in Fig. 3. As shown in Fig. 3, after adding to HCPs, the elongation at breaking of HCPs/PI MMMs gradually decreases with increasing amounts of HCPs. However, the elongation at breaking of HCPs/PI MMMs is higher than that of pure PI film. However, the value of the elongation at breaking of pure PI film and HCPs/PI MMMs is not large, and the value of the elongation at breaking of the HCPs/PI0.02 is the highest (only $3.9 \%$ ). This may be because PI has high rigidity, and molecular chain motion is difficult. The tensile strength of HCPs/PI MMMs increases in the beginning and then decreases. The tensile strength of HCPs/PI MMMs reaches up to the maximum about $44.2 \mathrm{MPa}$ when the amount of HCPs is 0.04 wt.\%. HCPs are a kind of pure organic fillers. They have good compatibility with $\mathrm{Pl}$, and therefore HCPs/PI MMMs have no defects, and their tensile strength does not decrease. In addition, due to the presence of 2-phenylimidazole in HCPs and hydrogen bonds between the $\mathrm{N}-\mathrm{H}$ bond on the imidazole ring and $\mathrm{C}=0$ bond in $\mathrm{PI}$, they can increase the tensile strength of $\mathrm{HCPs} / \mathrm{PI}$ MMMs due to incorporation of HCPs fillers; the result is similar to previous reports in which filler can enhance the mechanical strength of MMMs[46, 47]. The tensile strength of HCPs/PI MMMs will reduce because of excessive HCPs that may be related to the interfacial effect forming agglomeration of HCPs.

\subsection{Gas separation performance}

Gas separation performance is an important and ultimate index to evaluate the quality of MMMs. Fig. 4a shows gas separation performance of pure PI film and $\mathrm{HCPs} / \mathrm{PI} \mathrm{MMMs}$ for $\mathrm{CO}_{2} / \mathrm{CH}_{4}$ at $35^{\circ} \mathrm{C}$ and $0.1 \mathrm{MPa}$ of feed gas pressure. The permeation flux of pure $\mathrm{PI}$ film toward $\mathrm{CO}_{2}$ is $62.41 \mathrm{barrer}$, and the ideal selectivity to $\mathrm{CO}_{2} / \mathrm{CH}_{4}$ is 20.76. Simultaneously, as the amount of HCPs varies from $0.02 \mathrm{wt} \%$ to 0.08 wt.\%, the permeability of HCPs/PI MMMs to $\mathrm{CO}_{2}$ is 63.36 barrer, 105.85 barrer, 86.22 barrer, and 68.58 barrer. It increases at first and then decreases. Moreover, the permeability of all HCPs/PI MMMs is higher than that of pure PI film. When the amount of HCPs is $0.04 \mathrm{wt} . \%$, the permeability of HCPs/PI MMMs to $\mathrm{CH}_{4}$ and $\mathrm{CO}_{2}$ enhances $125 \%$ and $69.6 \%$, respectively. The results demonstrate that $\mathrm{HCPs} / \mathrm{PI}$ MMMs facilitate $\mathrm{CH}_{4}$ and $\mathrm{CO}_{2}$ transport. Besides, $\mathrm{HCPs} / \mathrm{PI}$ MMMs have better transport than pure PI film. 
Fortunately, the ideal selectivity is $14.57,15.67,14.66$, and 13.59 . The ideal selectivity of all HCPs/PI MMMs only decreases slightly than a pure PI film.

To better assess gas transport properties of $\mathrm{MMMs}$, the $\mathrm{CO}_{2}$ and $\mathrm{CH}_{4}$ permeability and ideal selectivity data are displayed in Fig. $4 \mathrm{~b}$ in a Robeson's diagram for the corresponding $\mathrm{CO}_{2}$ and $\mathrm{CH}_{4}$ pair. The ideal selectivity obviously decreases as Robeson defines the linear relationship as "upper bound" accompanied by an improvement of gas permeability in MMMs. Here, the $\mathrm{CO}_{2}$ and $\mathrm{CH}_{4}$ are below the upper-bound line and represent the perfect combination of permeability and ideal selectivity for the fixed gas pair. However, they show a favorable tendency to increase the gas permeability, while the ideal selectivity basically remains unchanged after adding HCPs, which is different from the typical trade-off phenomenon of polymer membranes. In this respect, the HCPs are used to prepare MMMs in an effective approach to successfully improve gas transport and break the trade-off effect.

Next, $\mathrm{O}_{2}$ and $\mathrm{N}_{2}$ permeability experiments were carried out to prove that HCPs can promote different gas transport. The $\mathrm{O}_{2} / \mathrm{N}_{2}$ separation performance of PI film and different HCPs/PI MMMs and Robenson's upper bound correlation (2008)[48] for $\mathrm{O}_{2} / \mathrm{N}_{2}$ separation are shown in Fig. 5. Fig. 5a shows the gas separation performance of pure PI film and HCPs/PI MMMs for $\mathrm{O}_{2} / \mathrm{N}_{2}$. According to Fig. 5 a, the pristine PI film exhibits that the permeability of $\mathrm{O}_{2}$ is 13.85 barrer, and the ideal selectivity of $\mathrm{O}_{2} / \mathrm{N}_{2}$ pair is 3.29 . Meanwhile, in terms of HCPS/PI MMMs, the permeability of HCPs/PI MMMs to $\mathrm{O}_{2}$ increases at first and then decreases as the amount of HCPs changes from $0.02 \mathrm{wt} . \%$ to $0.08 \mathrm{wt} . \%$. Moreover, the permeability of the HCPs/PI MMMs to $\mathrm{O}_{2}$ is higher than that of pristine PI film. When the HCPs were $0.04 \mathrm{wt} \%$, the permeability of the HCPs/PI MMMs to $\mathrm{O}_{2}$ is 24.03 barrer and plateaus. Compared with the pristine PI film, the permeability of HCPs/PI MMMs toward $\mathrm{O}_{2}$ and $\mathrm{N}_{2}$ increases by $73.5 \%$ and $91.6 \%$, respectively, while the ideal selectivity of $\mathrm{HCPs} / \mathrm{PI}$ MMMs toward $\mathrm{O}_{2} / \mathrm{N}_{2}$ pair only reduces $9.7 \%$. The results demonstrate that $\mathrm{HCPs} / \mathrm{PI}$ MMMs also facilitate $\mathrm{O}_{2}$ and $\mathrm{N}_{2}$ transport, improve gas permeability, and maintain stable selectivity.

Obviously, as shown in Fig. 4a and Fig. 5a, HCPs/PI MMMs have relatively weaker transport abilities to $\mathrm{CH}_{4}, \mathrm{O}_{2}$, and $\mathrm{N}_{2}$ than $\mathrm{CO}_{2}$. $\mathrm{HCPs}$ with imidazole-type poly-ionic liquid structures have certain catalytic properties for the cyclization of $\mathrm{CO}_{2}$ to carbonate. These can be used as a $\mathrm{CO}_{2}$ capture and conversion material[49-52]. This makes $\mathrm{CO}_{2}$ easy to be adsorbed in HCPs. When HCPs are used as the filler of PI MMMs, the solubility coefficient of PI MMMs to $\mathrm{CO}_{2}$ can be enhanced, and it may increase the gas permeability and accelerate the $\mathrm{CO}_{2}$ gas transport.

The results on permeability and ideal selectivity in the Robeson's diagram for the corresponding $\mathrm{O}_{2}$ and $\mathrm{N}_{2}$ pair (Fig. 5b) are analogous to the corresponding $\mathrm{CO}_{2}$ and $\mathrm{CH}_{4}$ pair; however, the permeability and ideal selectivity of HCPs/PI MMMs to $\mathrm{O}_{2} / \mathrm{N}_{2}$ are obviously lower than those of $\mathrm{CO}_{2} / \mathrm{CH}_{4}$. This also demonstrates that the interaction between $\mathrm{HCPs}$ and $\mathrm{CO}_{2}$ leads to better permeability and selectivity than that of HCPs and $\mathrm{O}_{2}$. 


\subsection{Analysis of gas separation process}

The gas separation performance of MMMs strongly depends on MMM structures. To gain an insight into the relation between the permeability and selectivity of MMMs and microstructure, the average interspacing distance (d-spacing) stands for the distance between polymer chain segments that reflects the permeability coefficient of MMMs. The d-spacing value of MMMs can be calculated by Bragg's equation[28]. XRD patterns of the PI film and HCPs/PI MMMs are shown in Fig. 6. The results demonstrate that the PI film and the HCPs/PI MMMs have broad amorphous peaks that are attributed to the presence of bulky - $\mathrm{C}\left(\mathrm{CF}_{3}\right)_{2}$ - groups in Pl; thus, the PI film and HCPs/PI MMMs have loose chain packing[53].

Generally, the amorphous nature of $\mathrm{PI}$ is favorable to gas permeation. The gas permeability of polymer membranes will strengthen with increasing d-spacing. The d-spacing values of PI film and HCPs/PI MMMs are $5.58 \AA, 5.60 \AA, 5.70 \AA, 5.70 \AA$, and $5.46 \AA$ (Table S1). The results show that the d-spacing trend for the PI films and the HCPs/PI MMMs gradually increases and then reduces, which agrees with different gas permeability observations except for HCPs-0.06/PI MMMs. Although the d-spacing value of HCPs$0.04 / \mathrm{PI}$ MMMs is equal to that of HCPs-0.06/PI MMMs, the gas permeability of HCPs-0.04/PI MMMs is higher than that of HCPs-0.06/PI MMMs. It is very possible that the free spaces in the microstructure besides the $d$-spacing of polymer chain segments have a fundamental effect on the gas permeability of polymer membranes[45].

Fig. 7 shows the molecular simulation diagrams of the PI and HCPs/PI systems after MD simulation. In this simulation, Fig. $7 \mathrm{a}$ and $7 \mathrm{~b}$ are schematic representations of simulated molecular cells of the $\mathrm{PI}$ and HCPs/PI systems, in which twenty-three molecular chains composed of 6FDA-DAPI with 30 repeating units for the $\mathrm{PI}$ are placed in a cube box. The blue and gray parts indicate free volume and occupied volume, respectively. Fig. 7 shows that the free volume of HCPs/PI MMMs is significantly larger than that of pure PI films. According to the literature, the large free volume provides a fast channel for gas transport, thereby enhancing gas permeability[27, 45]. The other simulation results of MMMs containing different HCP amounts are shown in supporting information Fig. S2; the specific values obtained by simulation are shown in supporting information Table S1.

The density of HCPs/PI mixed polymers in stable state varied from 1.5358 to $1.3553 \mathrm{~g} / \mathrm{cm}^{3}$, which is higher than 6FDA-based polyimides, and the FFV of MMMs (0.1892-0.2498) is also higher than most of 6FDA-based polyimide[54]. Combining supporting information Fig. $S 2$ with Table $S 1$, we see that the FFV of MMMs containing different HCP amounts is larger than that of pure PI films. As the amount of HCPs increases, the FFV of PI MMMs first increases and then slightly decreases. Therefore, although the permeation flux of HCPs/PI MMMs to different gases is higher than that of pure PI films, the gas transport of HCPs/PI MMMs does not always increase with more HCPs. When the amount of HCPs added is $0.04 \%$, the FFV of HCPs/PI MMMs reaches a maximum (0.2498). Therefore, the permeability of different gases is the largest. The permeability gradually reduces as even more is added. This also proves that the gas permeability not only is related to the $d$-space of the membranes but also has a greater 
relationship with the FFV of the membrane. In contrast, the ideal selectivity of different gas pairs reduces according to the large FFV; this result agrees with Fig. 4 and Fig. 5.

\section{Conclusion}

In this work, HCPs/PI MMMs were successfully obtained by HCPs containing 2-phenylimidazole structure as a filler and 6-FDA-based PI as a polymer matrix. The as-prepared MMMs were characterized by XRD, FT-IR, SEM, and a universal tensile testing machine. The results show that the as-prepared HCPs/PI MMMs were amorphous with no chemical bonding between HCPs and PI. The HCPs could be easily dispersed in the PI matrix. Even at 0.08 wt.\% HCP, the MMMs still did not cause defects at the interface between HCPs and PI due to their perfect compatibility other than slight agglomeration.

The MMMs had good mechanical properties due to the structure of PI itself and the presence of HCPs. When the HCPs were $0.04 \mathrm{wt}$.\%, the tensile strength at break of MMMs was as high as $44.2 \mathrm{MPa}$, which is much greater than the 24.0 MPa of pure PI film. Gas permeation experiments showed that MMMs had better gas transport capabilities than pure PI films, especially for $\mathrm{CO}_{2}$; the permeability was improved, and the ideal selectivity is maintained without drastically decreasing. The permeability of $\mathrm{CO}_{2}$ and $\mathrm{O}_{2}$ was $69.6 \%$ and $73.5 \%$ higher than that of pure PI film, and the permeability of $\mathrm{CH}_{4}$ and $\mathrm{N}_{2}$ was $124.0 \%$ and $91.6 \%$ higher than that of pure PI film. However, the ideal selectivity of $\mathrm{MMMs}$ for $\mathrm{CO}_{2} / \mathrm{CH}_{4}$ and $\mathrm{O}_{2} / \mathrm{N}_{2}$ gas pairs was only reduced by about $24.5 \%$ and $9.7 \%$, respectively. The molecular dynamics simulations show that the permeability trend of MMMs agrees with the FFV change of MMMs. The HCPs used to fabricate MMMs have potential applications in the field of natural gas purification and $\mathrm{O}_{2}$ enrichment.

\section{Declarations}

\section{Acknowledgments}

This work was supported by the National Natural Science Foundation of China (No.51309074), the Natural Science Foundation of Hebei Province (No. B2016202271).

\section{Conflict of interest}

The authors declared that they have no conflict of interest.

\section{References}

1. Liu, Z., Liu, Y., Qiu, W. \& Koros, W. J. Molecularly Engineered 6FDA-Based Polyimide Membranes for Sour Natural Gas Separation. Angew. Chem. Int. Edit. 59, 2-9 https://doi.org/10.1002/anie.202003910 (2020). 
2. Iulianelli, A. \& Drioli, E. Membrane engineering: Latest advancements in gas separation and pretreatment processes, petrochemical industry and refinery, and future perspectives in emerging applications, Fuel Process. Technol. 206(2020) 106464.

https://doi.org/10.1016/j.fuproc.2020.106464

3. Liu, G. et al. Molecularly Designed Stabilized Asymmetric Hollow Fiber Membranes for Aggressive Natural Gas Separation. Angew. Chem. Int. Edit. 55, 13754-13758 (2016). https://doi.org/10.1002/ anie. 201607055

4. Zhang, W., Cheng, Y., Guo, C., Xie, C. \& Xiang, Z. Cobalt Incorporated Porous Aromatic Framework for C02/CH4 Separation. Ind. Eng. Chem. Res. 57, 10985-10991 https://doi.org/10.1021/acs.iecr.8b01874 (2018).

5. Chen, K. K., Salim, W., Han, Y., Wu, D. \& Ho, W. S. W. Fabrication and scale-up of multi-leaf spiralwound membrane modules for CO2 capture from flue gas. J. Membr. Sci. 595, 117504 https://doi.org/10.1016/j.memsci.2019.117504 (2020).

6. Dong, S., Wang, Z., Sheng, M., Qiao, Z. \& Wang, J. Scaling up of defect-free flat membrane with ultrahigh gas permeance used for intermediate layer of multi-layer composite membrane and oxygen enrichment. Sep. Purif. Technol. 239, 116580 https://doi.org/10.1016/j.seppur.2020.116580 (2020).

7. Matsumiya, N., Teramoto, M., Kitada, S. \& Matsuyama, H. Evaluation of energy consumption for separation of $\mathrm{CO} 2$ in flue gas by hollow fiber facilitated transport membrane module with permeation of amine solution. Sep. Purif. Technol. 46, 26-32 https://doi.org/10.1016/j.seppur.2005.04.006 (2005).

8. Wang, S. et al. Advances in high permeability polymer-based membrane materials for $\mathrm{CO} 2$ separations. Energ. Environ. Sci. 9, 1863-1890 (2016). https://doi.org/10.1039/ C6EE00811A

9. Pérez-Francisco, J. M. et al. CMS membranes from PBI/PI blends: Temperature effect on gas transport and separation performance. J. Membr. Sci. 597, 117703 https://doi.org/10.1016/j.memsci.2019.117703 (2020).

10. Moghadam, F., Kamio, E., Yoshioka, T. \& Matsuyama, H. New approach for the fabrication of doublenetwork ion-gel membranes with high C02/N2 separation performance based on facilitated transport. J. Membr. Sci. 530, 166-175 https://doi.org/10.1016/j.memsci.2017.02.032 (2017).

11. Wu, H. et al. Facilitated transport mixed matrix membranes incorporated with amine functionalized MCM-41 for enhanced gas separation properties. J. Membr. Sci. 465, 78-90 (2014). https://doi.org/ 10.1016/j.memsci.2014.04.023

12. Sun, J. et al. MOF-801 incorporated PEBA mixed-matrix composite membranes for CO2 capture. Sep. Purif. Technol. 217, 229-239 https://doi.org/10.1016/j.seppur.2019.02.036 (2019).

13. Duan, K., Wang, J., Zhang, Y. \& Liu, J. Covalent organic frameworks (COFs) functionalized mixed matrix membrane for effective C02/N2 separation. J. Membr. Sci. 572, 588-595 https://doi.org/10.1016/j.memsci.2018.11.054 (2019).

14. Nik, O. G., Chen, X. Y. \& Kaliaguine, S. Functionalized metal organic framework-polyimide mixed matrix membranes for CO2/CH4 separation. J. Membr. Sci. 413-414 
https://doi.org/10.1016/j.memsci.2012.04.003 (2012).

15. Farrokhara, M. \& Dorosti, F. New high permeable polysulfone/ionic liquid membrane for gas separation. Chinese J. Chem. Eng. https://doi.org/10.1016/j.cjche. 2020.04 .002 (2020).

16. Ishaq, S., Tamime, R., Bilad, M. R. \& Khan, A. L. Mixed matrix membranes comprising of polysulfone and microporous Bio-MOF-1: Preparation and gas separation properties. Sep. Purif. Technol. 210, 442-451 https://doi.org/10.1016/j.seppur.2018.08.031 (2019).

17. Mubashir, M., Yin fong, Y., Leng, C. T., Keong, L. K. \& Jusoh, N. Study on the effect of process parameters on $\mathrm{CO} 2 / \mathrm{CH} 4$ binary gas separation performance over $\mathrm{NH} 2-\mathrm{MIL}-53(\mathrm{Al}) /$ cellulose acetate hollow fiber mixed matrix membrane. Polym. Test. 81, 106223 https://doi.org/10.1016/j.polymertesting.2019.106223 (2020).

18. Hou, T. et al. Cellulose membranes with polyethylenimine-modified graphene oxide and zinc ions for promoted gas separation. Cellulose. 27, 3277-3286 https://doi.org/10.1007/s10570-019-02962-4 (2020).

19. Ma, X., Abdulhamid, M., Miao, X. \& Pinnau, I. Facile Synthesis of a Hydroxyl-Functionalized Tröger's Base Diamine: A New Building Block for High-Performance Polyimide Gas Separation Membranes. Macromolecules. 50, 9569-9576 https://doi.org/10.1021/acs.macromol.7b02301 (2017).

20. Abdulhamid, M. A., Ma, X., Miao, X. \& Pinnau, I. Synthesis and characterization of a microporous 6FDA-polyimide made from a novel carbocyclic pseudo Tröger's base diamine: Effect of bicyclic bridge on gas transport properties. Polymer. 130, 182-190 https://doi.org/10.1016/j.polymer.2017.10.017 (2017).

21. Swaidan, R., Ghanem, B., Al-Saeedi, M., Litwiller, E. \& Pinnau, I. Role of Intrachain Rigidity in the Plasticization of Intrinsically Microporous Triptycene-Based Polyimide Membranes in Mixed-Gas C02/CH4 Separations. Macromolecules. 47, 7453-7462 https://doi.org/10.1021/ma501798v (2014).

22. Sanaeepur, H. et al. Polyimides in membrane gas separation: Monomer's molecular design and structural engineering. Prog. Polym. Sci. 91, 80-125 (2019). https://doi.org/10.1016/ j.progpolymsci.2019.02.001

23. K.a.W, A. M. Decarboxylation-Induced Cross-Linking of a Polyimide for Enhanced CO2 Plasticization Resistance. Macromolecules. 41, 7920-7927 https://doi.org/10.1021/ma801586f (2008).

24. Cho, Y. J. \& Park, H. B. High performance polyimide with high internal free volume elements. Macromol. Rapid. Commun. 32, 579-586 (2011). https://doi.org/10.1002/marc. 201000690

25. Bee Ting Low, T. S. C., Chen, H., Jean, Y. C., Kumari, P. \& Pramoda Tuning the Free Volume Cavities of Polyimide Membranes via theConstruction of Pseudo-Interpenetrating Networks for Enhanced Gas Separation Performancemace. Macromolecules. 42, 7042-7054 https://doi.org/10.1021/ma901251y (2009).

26. Liu, G. et al. Zeolite-like MOF nanocrystals incorporated 6FDA-polyimide mixed-matrix membranes for $\mathrm{CO} 2 / \mathrm{CH} 4$ separation. J. Membr. Sci. 565, 186-193 https://doi.org/10.1016/j.memsci.2018.08.031 (2018). 
27. Norwahyu Jusoh, Y. F. Y., Lau, K. K. \& Shariff, A. M. Enhanced gas separation performance using mixed matrix membranes containing zeolite T and 6FDA-durene polyimide. J. Membr. Sci. 525, 175186 (2017). https://doi.org/10.1016/j.memsci. 2016.10.044

28. Japip, S., Wang, H., Xiao, Y., Shung, T. \& Chung Highly permeable zeolitic imidazolate framework (ZIF)-71 nano-particles enhanced polyimide membranes for gas separation. J. Membr. Sci. 467, 162174 https://doi.org/10.1016/j.memsci.2014.05.025 (2014).

29. Hu, C. C. et al. Separation behavior of amorphous amino-modified silica nanoparticle/polyimide mixed matrix membranes for gas separation. J. Membr. Sci. 595, 117542 https://doi.org/10.1016/j.memsci.2019.117542 (2020).

30. Kang, Z. et al. Mixed Matrix Membranes (MMMs) Comprising Exfoliated 2D Covalent Organic Frameworks (COFs) for Efficient CO2 Separation. Chem. Mater. 28, 1277-1285 https://doi.org/10.1021/acs.chemmater.5b02902 (2016).

31. Rodenas, T. et al. Metal-organic framework nanosheets in polymer composite materials for gas separation. Nat. Mater. 14, 48-55 https://doi.org/10.1038/nmat4113 (2015).

32. Rodenas, T. et al. Visualizing MOF Mixed Matrix Membranes at the Nanoscale: Towards StructurePerformance Relationships in C02/CH4 Separation Over NH2-MIL-53(Al)@PI. Adv. Funct. Mater. 24, 249-256 https://doi.org/10.1002/adfm.201203462 (2014).

33. Fan, Y. et al. Zn(II)-modified imidazole containing polyimide/ZIF-8 mixed matrix membranes for gas separations. J. Membr. Sci. 597, 117775 https://doi.org/10.1016/j.memsci.2019.117775 (2020).

34. Ahmad, M. Z., Martin-Gil, V., Perfilov, V., Sysel, P. \& Fila, V. Investigation of a new co-polyimide, 6FDAbisP and its ZIF-8 mixed matrix membranes for $\mathrm{CO} 2 / \mathrm{CH} 4$ separation. Sep. Purif. Technol. 207, 523534 (2018). https://doi.org/10.1016/j.seppur. 2018.06.067

35. Lin, R., Ge, L., Liu, S., Rudolph, V. \& Zhu, Z. Mixed-Matrix Membranes with Metal-Organic FrameworkDecorated CNT Fillers for Efficient CO2 Separation. ACS Appl. Mater. Inter. 7, 14750-14757 https://doi.org/10.1021/acsami.5b02680 (2015).

36. Lin, R., Ge, L., Diao, H., Rudolph, V. \& Zhu, Z. Propylene/propane selective mixed matrix membranes with grape-branched MOF/CNT filler. J. Mater. Chem. A. 4, 6084-6090 https://doi.org/10.1039/c5ta10553f (2016).

37. Cheng, Y. et al. Enhanced Polymer Crystallinity in Mixed-Matrix Membranes Induced by Metal-Organic Framework Nanosheets for Efficient CO2 Capture. ACS Appl. Mater. Inter. 10, 43095-43103 https://doi.org/10.1021/acsami.8b16386 (2018).

38. Tan, L. \& Tan, B. Hypercrosslinked porous polymer materials: design, synthesis, and applications. Chem. Soc. Rev. 46, 3322-3356 https://doi.org/10.1039/c6cs00851h (2017).

39. Lau, C. H. et al. Hypercrosslinked Additives for Ageless Gas-Separation Membranes. Angew. Chem. Int. Edit. 55, 1998-2001 https://doi.org/10.1002/anie.201508070 (2016).

40. Mondal, J. et al. Fabrication of Ruthenium Nanoparticles in Porous Organic Polymers: Towards Advanced Heterogeneous Catalytic Nanoreactors, Chem-Eur. J. 21, 19016-19027 https://doi.org/10.1002/chem.201504055 (2015). 
41. Wang, K. et al. Acid and base coexisted heterogeneous catalysts supported on hypercrosslinked polymers for one-pot cascade reactions. J. Catal. 348, 168-176 https://doi.org/10.1016/j.jcat.2017.02.024 (2017).

42. Dose, M. E. et al. Thermally cross-linked diaminophenylindane (DAPI) containing polyimides for membrane based gas separations. Polymer. 161, 16-26 (2019). https://doi.org/10.1016/ j.polymer.2018.11.050

43. Álvarez, C., Lozano, Ã. E., Juan-y-Seva, M. \& de la Campa, J. G. Gas separation properties of aromatic polyimides with bulky groups. Comparison of experimental and simulated results. J. Membr. Sci. 602, 117959 (2020). https://doi.org/10.1016/j.memsci. 2020.117959

44. Jun, H. C. et al. Membrane Hollow Fibers of Cu3(BTC)2 MOF and Polyimide for Gas Separation and Adsorption. Ind. Eng. Chem. Res. 49, 12605-12612 https://doi.org/10.1021/ie1014958 (2010).

45. Tong, H. et al. Preparation of fluorinated polyimides with bulky structure and their gas separation performance correlated with microstructure. Polymer. 69, 138-147 (2015). https://doi.org/10.1016/j.polymer. 2015.05.045

46. Li, W., Samarasinghe, S. A. S. C. \& Bae, T. H. Enhancing $\mathrm{CO} 2 / \mathrm{CH} 4$ separation performance and mechanical strength of mixed-matrix membrane via combined use of graphene oxide and ZIF-8. J. Ind. Eng. Chem. 67, 156-163 https://doi.org/10.1016/j.jiec.2018.06.026 (2018).

47. Li, H. et al. Simultaneous enhancement of mechanical properties and CO2 selectivity of ZIF-8 mixed matrix membranes: Interfacial toughening effect of ionic liquid. J. Membr. Sci. 511, 130-142 https://doi.org/10.1016/j.memsci.2016.03.050 (2016).

48. Robeson, L. M. The upper bound revisited. J. Membr. Sci. 320, 390-400 https://doi.org/10.1016/j.memsci.2008.04.030 (2008).

49. Sang, Y. \& Huang, J. Benzimidazole-based hyper-cross-linked poly(ionic liquid)s for efficient CO2 capture and conversion. Chem. Eng. J. 385, 123973 (2020). https://doi.org/ 10.1016/j.cej.2019.123973

50. Jadhav, A. H. et al. Effect of anion type of imidazolium based polymer supported ionic liquids on the solvent free synthesis of cycloaddition of CO2 into epoxide. Catal. Today. 265, 56-67 (2016). https://doi.org/ 10.1016/j.cattod.2015.09.048

51. Zhao, Y., Yao, C., Chen, G. \& Yuan, Q. Highly efficient synthesis of cyclic carbonate with CO2catalyzed by ionic liquid in a microreactor. Green Chem. 15, 446-452 https://doi.org/10.1039/c2gc36612f (2013).

52. Buyukcakir, O. et al. Porous cationic polymers: the impact of counteranions and charges on $\mathrm{CO} 2$ capture and conversion. Chem. Commun. 52, 934-937 https://doi.org/10.1039/C5CC08132G (2016).

53. Butnaru, I. et al. Insights into molecular engineering of membranes based on fluorinated polyimidepolyamide miscible blends which do not obey the trade-off rule. Sep. Purif. Technol. 233, 116031 https://doi.org/10.1016/j.seppur.2019.116031 (2020). 
54. Bas, C., Mercier, R., Dauwe, C. \& Albérola, N. D. Microstructural parameters controlling gas permeability and permselectivity in polyimide membranes. J. Membr. Sci. 349, 25-34 https://doi.org/10.1016/j.memsci.2009.11.016 (2010).

\section{Figures}

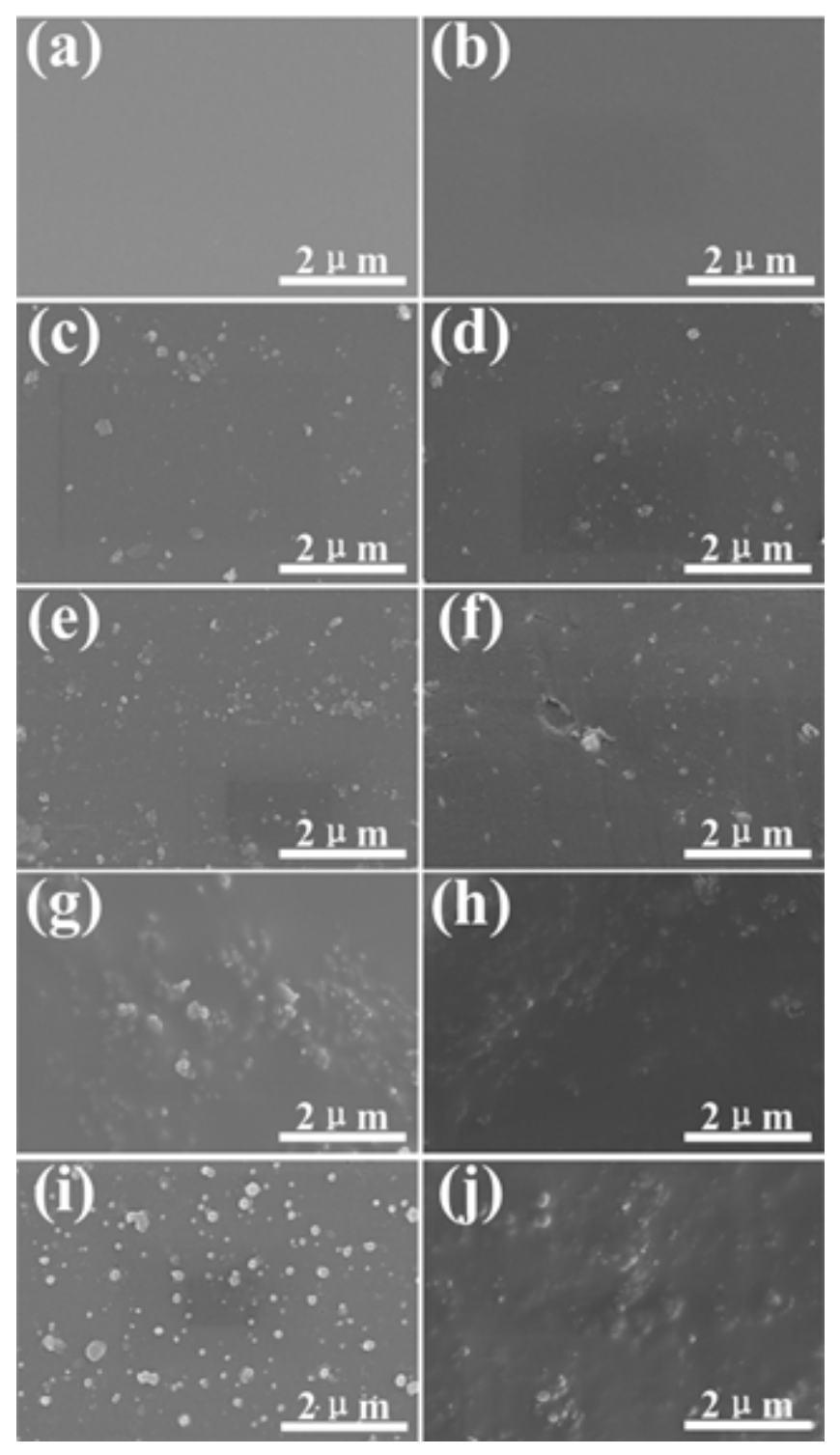

Figure 1

SEM images of PI film and PI/HCPs MMMs, (a) surface of PI film, (b) section of PI film, (c) surface of HCPs-0.02/PI MMMs, (d) section of HCPs-0.02/PI MMMs, (e) surface of HCPs-0.04/PI MMMs, (f) section of HCPs-0.04/PI MMMs, (g) surface of HCPs-0.06/PI MMMs, (h) section of HCPs-0.06/PI MMMs, (i) surface of HCPs-0.08/PI MMMs, and (j) section of HCPs-0.08/PI MMMs. 


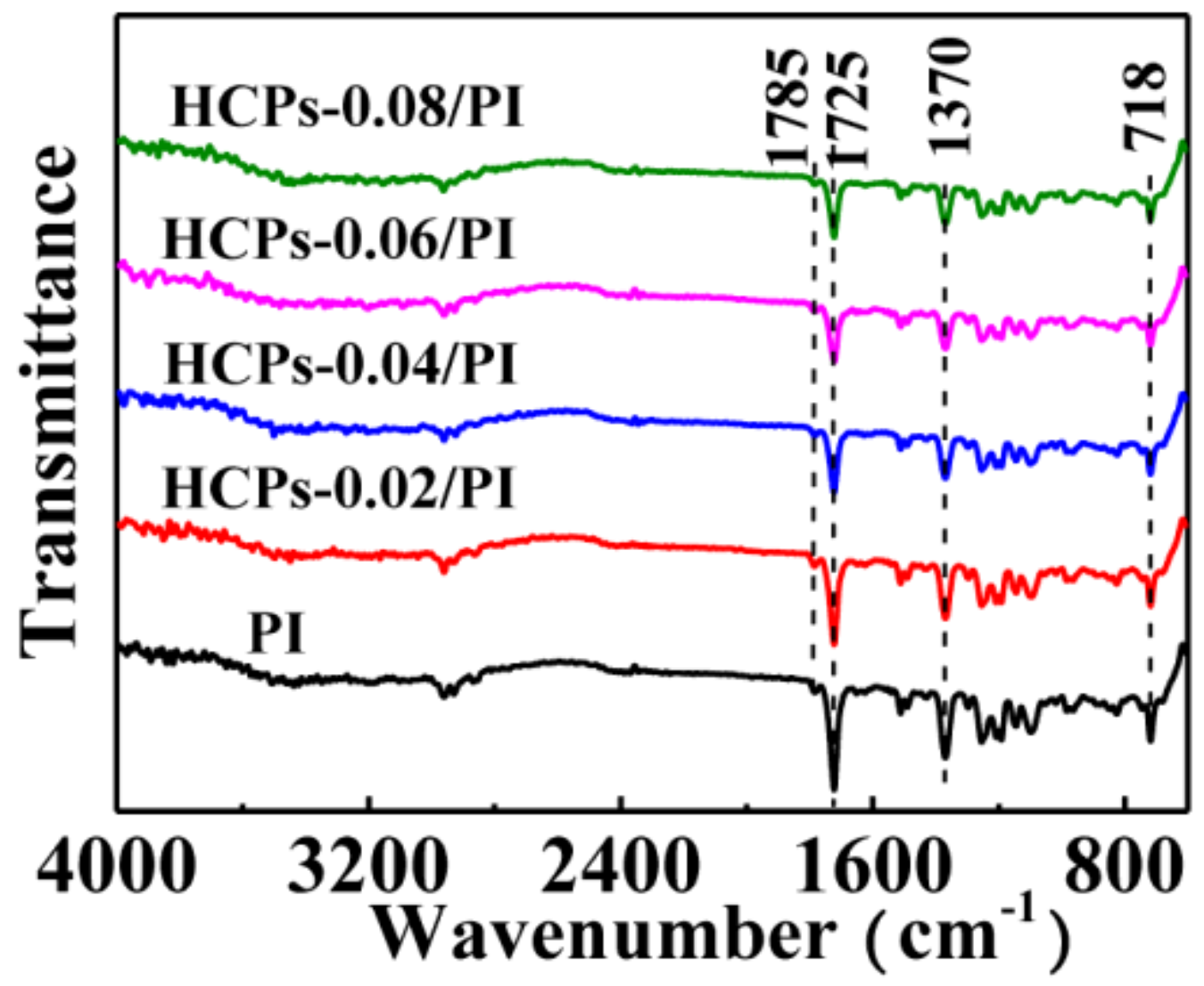

Figure 2

FT-IR spectra of pure PI film and different HCPs/PI MMMs.

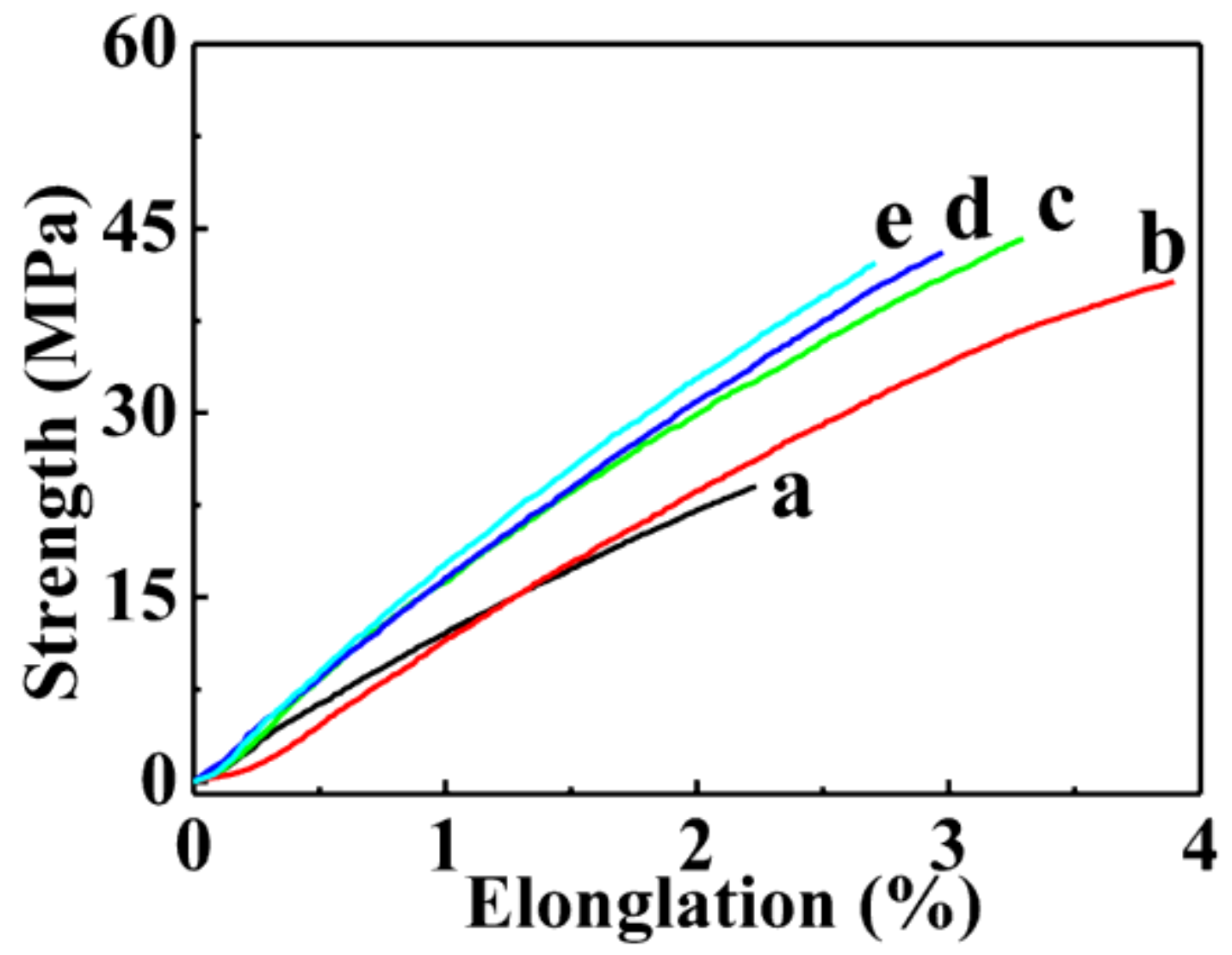


Figure 3

Elongation at break and tensile strength of different membranes (a) PI film, (b) HCPs-0.02/PI MMMs, (c) HCPs-0.04/PI MMMs, (d) HCPs-0.06/PI MMMs, and (e) HCPs-0.08/PI MMMs.
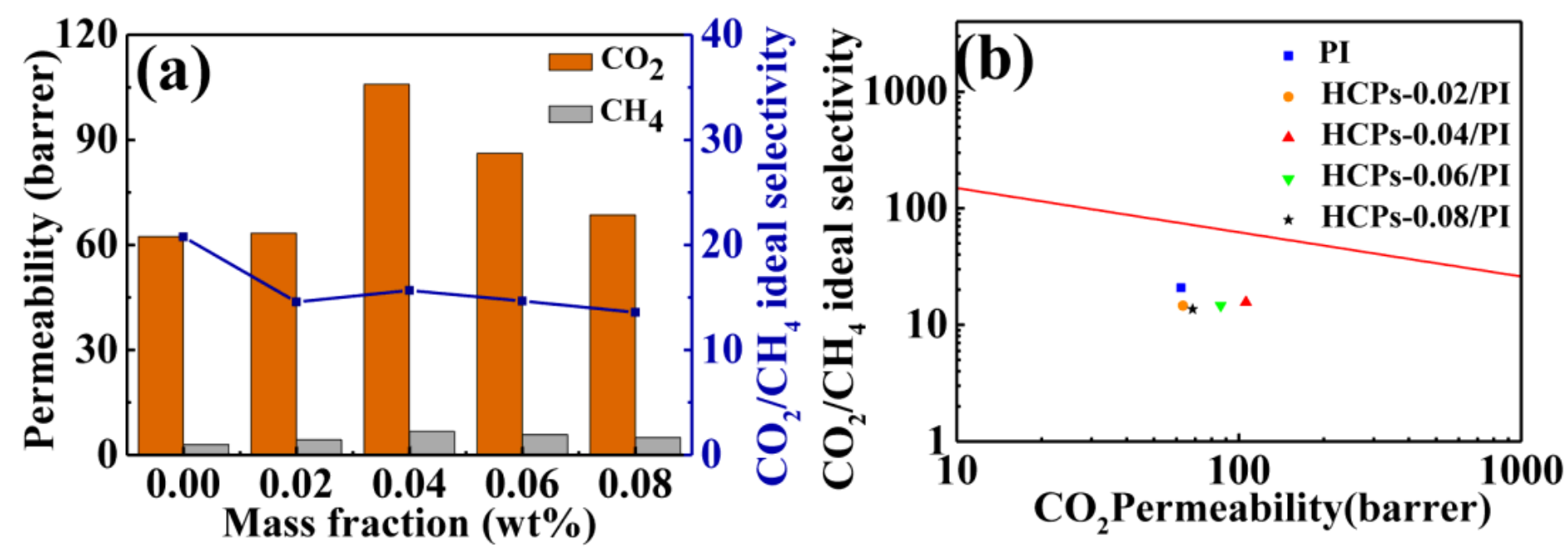

Figure 4

(a) $\mathrm{CO} 2 / \mathrm{CH} 4$ separation performance of PI film and different HCPs/PI MMMs and (b) upper bound correlation (2008) for $\mathrm{CO} 2 / \mathrm{CH} 4$ separation.
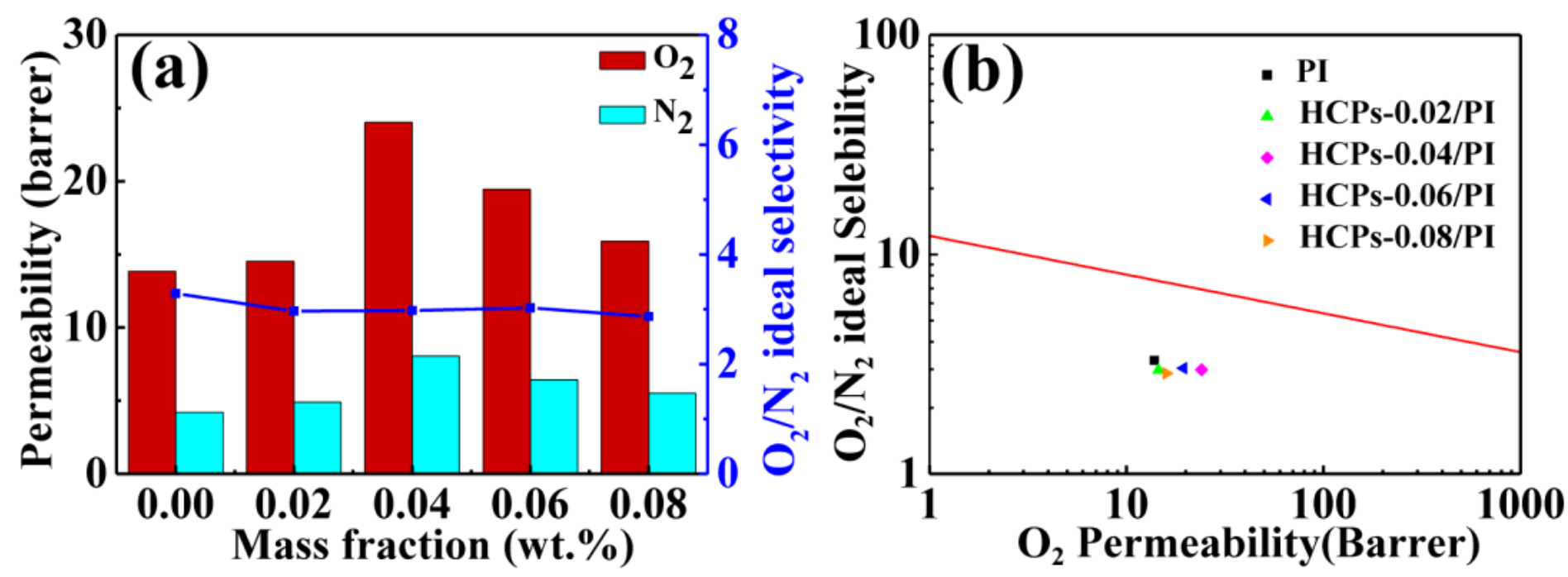

Figure 5

(a) 02/N2 separation performance of PI film and different HCPs/PI MMMs and (b) Robenson's upper bound correlation (2008) for O2/N2 separation. 


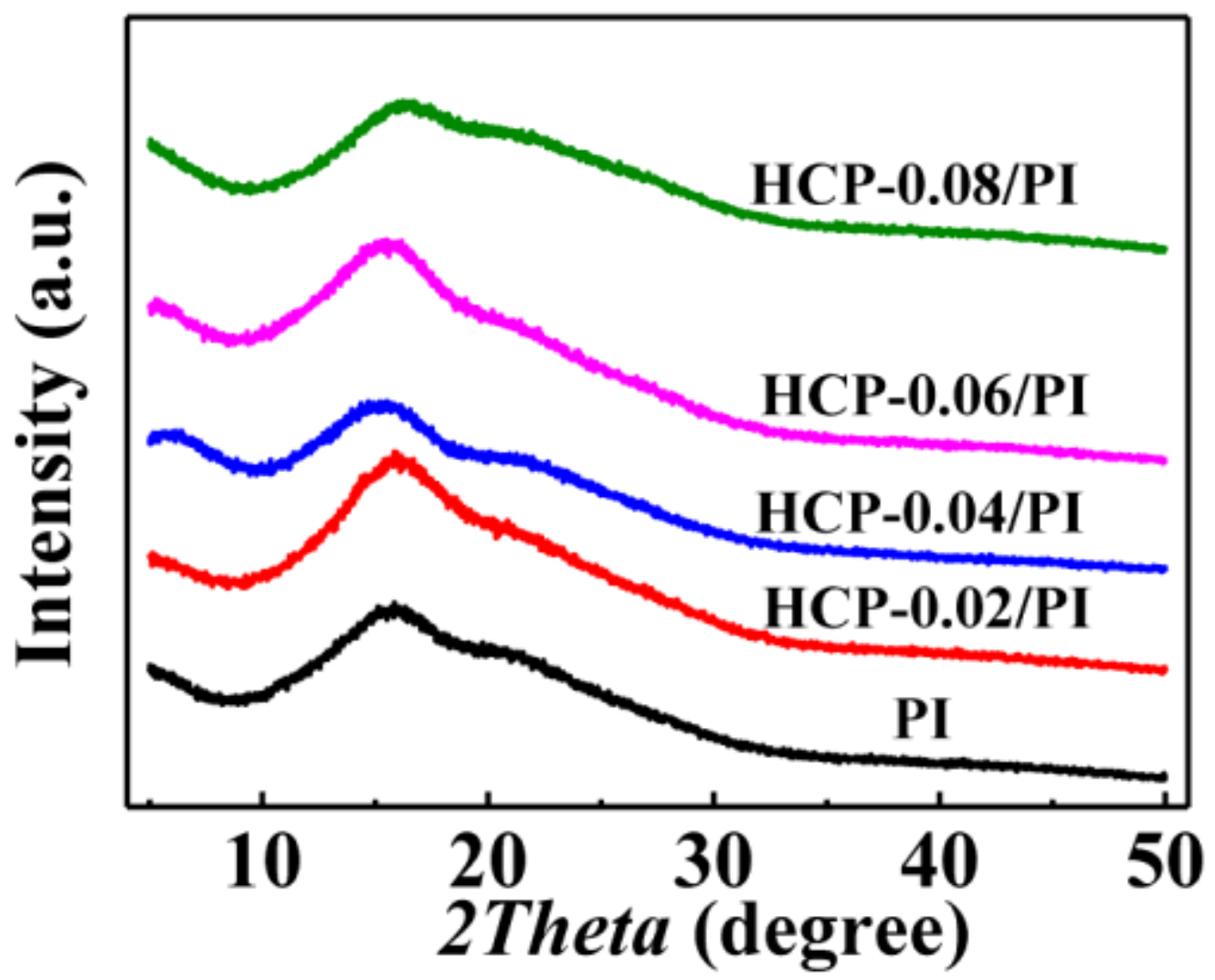

Figure 6

XRD patterns of the pure PI film and the HCPs/PI MMMs.
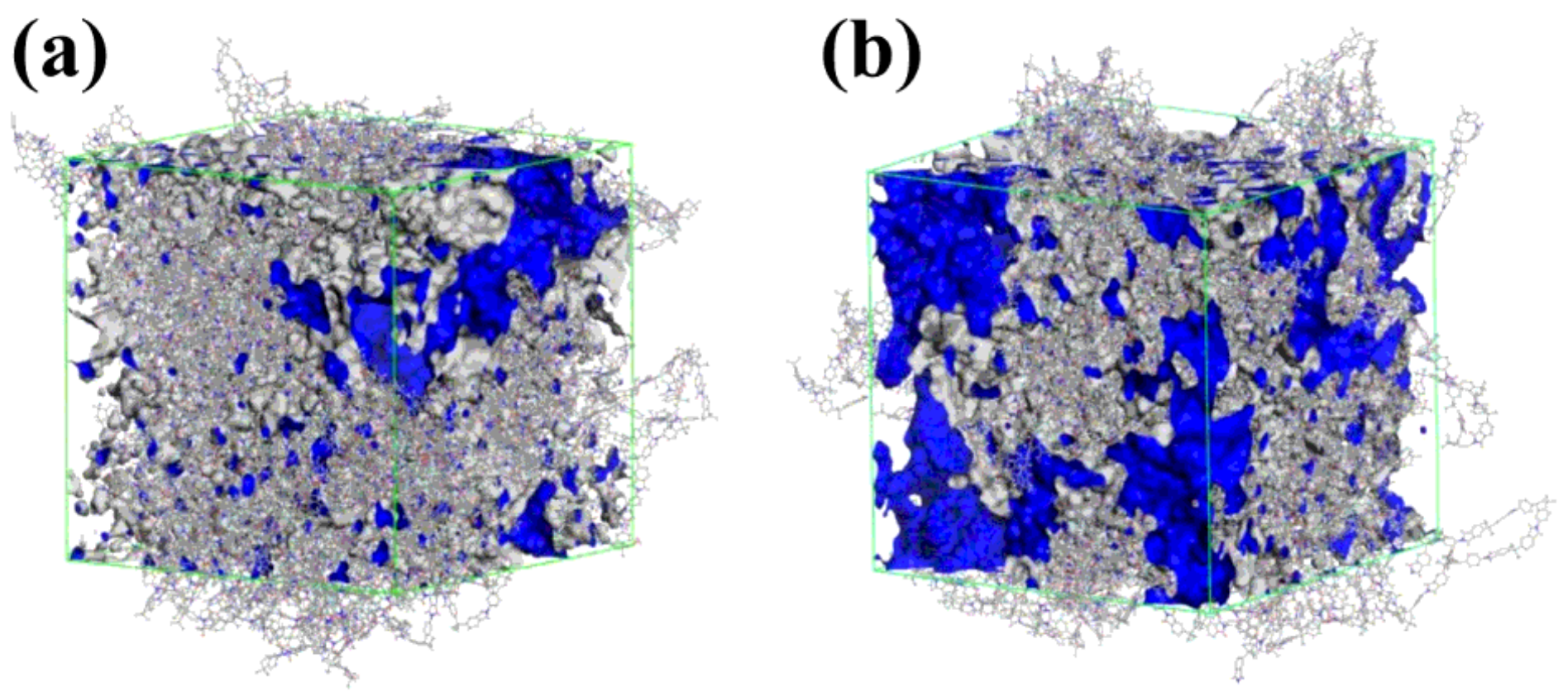

Figure 7

Illustrations on molecular dynamic simulations of (a) pure PI film and (b) HCPs-0.04/PI MMMs. 

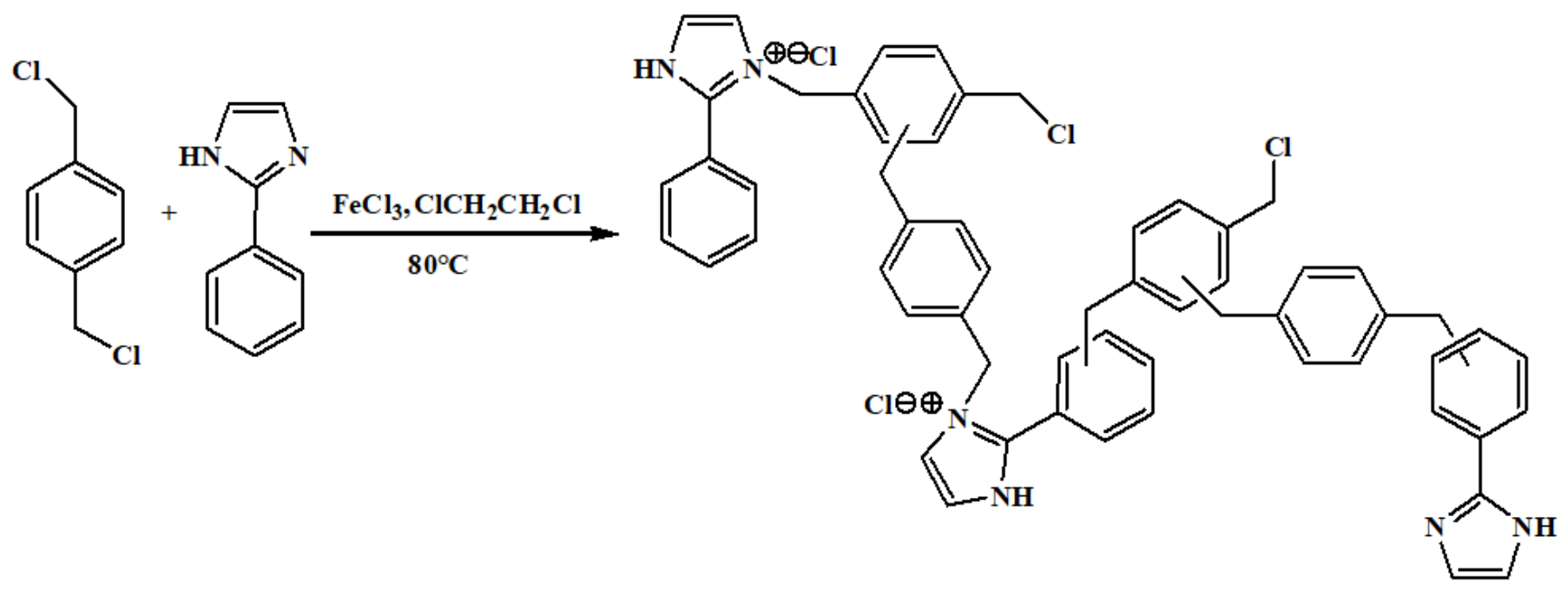

Figure 8

Synthesis of hyper-crosslinked polymers.

\section{Supplementary Files}

This is a list of supplementary files associated with this preprint. Click to download.

- Supportinformationfinal.docx 